\title{
Exposure and Risk Assessment of Operators to Insecticide Acetamiprid during Treatment on Apple Orchard
}

\author{
Eunhye Kim¹, Joon-Kwan Moon², Hyeri Lee ${ }^{1}$, Suhee Kim ${ }^{1}$, Yon-Jin Hwang ${ }^{1}$, \\ Byung-Joon Kim, Jinbeum Lee ${ }^{1}$, Dong-Hyuk Lee ${ }^{3}$, and Jeong-Han Kim ${ }^{1 *}$ \\ ${ }^{I}$ Department of Agricultural Biotechnology, Seoul National University, Seoul 151-742, Korea \\ ${ }^{2}$ Department of Plant Life and Environmental Sciences, Hankyong National University, Anseong 456-749, Korea \\ ${ }^{3}$ Apple Research Station, National Institute of Horticultural \& Herbal Science, Gunwie 716-812, Korea
}

\begin{abstract}
Occupational exposure and risk assessment were conducted to evaluate the safety of operators when insecticide acetamiprid was applied to apple orchard using a speed sprayer. Dermal patches, cotton gloves, socks, and masks were used to monitor the dermal exposure, and personal air pump with solid sorbent was used to measure the potential inhalation exposure. In validation to analytical methods, the limit of detection and limit of quantitation were $0.25 \mathrm{ng}$ and $1 \mathrm{ng}$, respectively. Good reproducibility (coefficient variation < $4 \%$ ), linearity (coefficient determination $>0.999$ ), and recovery $(85.3-118.2 \%)$ were obtained. Trapping efficiency of solid sorbent was $96.4 \%$ while breakthrough did not occur. Only hand exposure was measured on the gloves during mixing/loading to give 33-1,132 $\mu \mathrm{g}$. Exposure amount of operator 3 among 4 workers was noticeably high. The total volumes of spray liquid for operators were $535-1,235 \mathrm{~mL} \cdot \mathrm{h}^{-1}$, corresponding to $0.03-0.08 \%$ of the applied spray solution. Highest contaminated parts of body were thighs, chest, and lower legs. The inhalation exposure ratio to the total application amount was significantly low. However, wind seemed to affect the inhalation exposure of operator. For risk assessment, margin of safety was calculated by the application of cloth and dermal penetration rate to obtain values of much larger than 1 in all cases. Therefore, health risk of operators during treatment of acetamiprid in apple orchard could be of least possibility.
\end{abstract}

Additional key words: dermal exposure, inhalation exposure, margin of safety

\section{Introduction}

Apple is one of the most produced and consumed fruits in Korea. The cultivated area and amount of production is 31,167 ha and 379,541 ton in 2011, respectively (KOSIS, 2011). Pesticides are indispensible chemicals that are widely used to control insects, diseases, and weeds in agricultural fields, and 165 pesticides have been registered for apple (KFDA, 2012). Operators, who handle and apply pesticides in fields, can be exposed to pesticides during mixing/loading and spraying in apple orchard. Safety and health of operators during mixing/loading and application is a major concerned in agricultural activities (Ramos et al., 2010) because direct contact with pesticides by operators who handle and apply these agents can lead to harmful effects depending on the toxicity of pesticides. In orchards, $64.6 \%$ of famers prefer to use speed sprayers than power sprayers for the application of pesticides
(Hong et al., 2007). They wear long-sleeve shirts and long trouser instead of protective garments while spraying. Therefore there could be significant dermal exposure to operators to result in the unintended field-poisoning. During spraying, the representative routes of human exposure are dermal deposition and inhalation (Kim et al., 2012). Potential dermal exposure (PDE), measured by analyzing the quantity of a pesticide that deposited on body regions, provides the information on the amount of exposure. However, PDE data by themselves cannot be used as a risk indicator because it must be related to acceptable exposure limit. For this purpose, the margin of safety (MOS) has been proposed as a useful risk indication (Machado-Neto, 2001) that relates the acceptable exposure to a pesticide with the mass absorbed by the body, which can be estimated from PDE. MOS value higher than 1 are considered as an indication of safe working condition.

Acetamiprid ((E)-N1-[(6-chloro-3-pyridyl)methyl]-N2-cyano-

\footnotetext{
*Corresponding author: kjh2404@snu.ac.kr

※ Received 6 November 2012; Revised 27 November 2012; Accepted 2 December 2012. This study was carried out with the support of 'Cooperative Research Program for Agricultural Science and Technology Development (PJ0074112012)', Rural Development Administration, Suwon, Korea.
} 
N1-methoylacetamidine) (Table 1), is a systemic neonicotinoid insecticide which is intended to control sucking insects on crops such as leafy vegetables, citrus fruits, pome fruits, grapes, cotton, cole crops, and ornamental plants. It has a low acute and chronic toxicity in mammals with no evidence of carcinogenicity, neurotoxicity endocrine disruption, or mutagenicity (Maŕ n et al., 2004).

However, there is no previous exposure and risk assessment of the operator to insecticide acetamiprid in apple orchard, to our knowledge. Thus, in this study, dermal and inhalation exposure amounts were measured during mixing/loading and application of acetamiprid after analytical methods were fully validated. Body parts of major exposure were identified and MOS for operator was calculated based on PDE to find out whether work condition is safe or not.

\section{Materials and Methods}

\section{Reagents and Materials}

Acetamiprid (99.9\%) of analytical standard grade was purchased from Sigma-Aldrich (St. Louis, MO, USA). All solvents were HPLC-grade, and were purchased from Fisher Scientific Korea Ltd. (Ansung, Korea). Stock solution $\left(100 \mu \mathrm{g} \cdot \mathrm{mL}^{-1}\right.$ ) was prepared in HPLC-grade acetonitrile. The working standard solutions were prepared by serial dilution of stock solution in acetonitrile. Acetamiprid wettable powder (WP) $(8 \%)$ was purchased from a pesticide vender for field study.

\section{Dermal Patches, Gloves, Socks, and Masks}

Dermal patches for dermal exposure measurement were made by putting cellulose paper (Whatman $17 \mathrm{CHR}, 46 \times 57 \mathrm{~cm}$, $1 \mathrm{~mm}$ thickness; Kent, UK) in a patch pocket $(10 \times 10 \mathrm{~cm})$ with circular exposure part $\left(50 \mathrm{~cm}^{2}\right)$. Safety pins were used to attach patches on protective garment (SP protective, KleenGuard, Yuhan-kimberly Korea Ltd., Seoul, Korea) of operator. Cotton masks (face exposure; $200 \mathrm{~cm}^{2}$ ), cotton socks (feet exposure), cotton gloves (hands exposure) were purchased from local markets.

\section{Personal Monitor and U-shaped Glass Tube}

A PAM consists of air pump (Gillian Model 224-PCXR7, MSA, Dong Ha Trading Co., Ltd., Seoul, Korea), glass fiber filter (37 mm, SKC, Eighty Four, PA, USA) in open-faced cassette (SKC), and solid sorbent $\left(\mathrm{ORBO}^{\mathrm{TM}} 609\right.$ Amberlite XAD-2 400/200 mg, Supelco, St. Louis, MO, USA). U-shaped glass tube for trapping efficiency test was manufactured by Dae-Jung Chemical (Daejeon, Korea).

\section{Experimental Sites}

All the field experiments were carried out in apple research institute orchards (Table 1). The unit size of the site for repetition was $0.2 \mathrm{ha}$. The apple tree height was $2.5-5 \mathrm{~m}$, and rows were separated by 4- $6 \mathrm{~m}$. During the experiment, ambient temperature was $9-24^{\circ} \mathrm{C}$ and relative humidity was $16-61 \%$. Wind velocity did not exceed $4 \mathrm{~m} \cdot \mathrm{s}^{-1}$ (Kester 3000, Nielsen-Kellerman, Boothwyn, PA, USA).

\section{Chromatographic Condition}

All chromatographic analysis were performed on a high performance liquid chromatography (Agilent 1100 HPLC; CA, USA) with an automatic injector, a diode array detector, and a C18 column $(250 \times 4.6 \mathrm{~mm}, 5 \mu \mathrm{m}$ particle; Shiseido, Kyoto, Japan) at $40^{\circ} \mathrm{C}$. A gradient system was employed for 15 min at a flow rate of $1.0 \mathrm{~mL} \cdot \mathrm{min}^{-1}$ with $\mathrm{A}$ (water): $\mathrm{B}$ (acetonitrile) as follows: 50:50 for initial $1 \mathrm{~min}$; 30:70 for 10 min; 0:100 for $12 \mathrm{~min}$, and 50:50 for final $15 \mathrm{~min}$. Elution of acetamiprid was monitored at $250 \mathrm{~nm}$.

Limit of Detection (LOD), Limit of Quantitation (LOQ), Reproducibility, and Linearity of Calibration Curve

Aliquots $(10 \mu \mathrm{L})$ of acetamiprid standard solution $(0.01-1.0$

Table 1. Field and meteorological conditions in apple orchard employed in this study.

\begin{tabular}{lcccc}
\hline \hline Applicator & Operator 1 & Operator 2 & Operator 3 & Operator 4 \\
\hline Tree growth stage & \multicolumn{2}{c}{ Before blossom stage } \\
Area (ha) & 0.2 & 0.2 & 0.2 & 0.2 \\
Tree age (years) & $4-30$ & $3-35$ & $3-26$ & $5-25$ \\
Tree height $(\mathrm{m})$ & $3-4$ & 3.5 & $2.5-4$ & $3-4$ \\
Inner row crop distance $(\mathrm{m})$ & $0.5-1$ & $1-2$ & $1-3$ & $1-4$ \\
Row distance $(\mathrm{m})$ & $4-5$ & 4 & $4-6$ & $4-6$ \\
Temperature $\left({ }^{\circ} \mathrm{C}\right)$ & $10-24$ & $9-21$ & $7-20$ & $9-24$ \\
Relative humidity $(\%)$ & $22-54$ & $21-61$ & $16-62$ & $19-50$ \\
Wind speed $\left(\mathrm{m} \cdot \mathrm{s}^{-1}\right)$ & $0-2$ & $0-4$ & $0-1$ & $0-1$ \\
Precipitation & & & None & \\
\hline
\end{tabular}


$\mu \mathrm{g} \cdot \mathrm{mL}^{-1}$ ) were analyzed for LOD determination before LOQ calculation. For reproducibility test, LOQ levels $(1,10,50)$ of acetamiprid standard solution were analyzed six times by using an HPLC to calculate the coefficient of variation (CV). Various acetamiprid standard solutions $\left(0.1-10 \mu \mathrm{g} \cdot \mathrm{mL}^{-1}\right)$ were analyzed for establishment of calibration curve. After 1 and 3 days of storage, the linearity (coefficient determination, $\mathrm{R}^{2}$ ) of the curve was investigated again.

\section{Trapping Efficiency and Breakthrough Tests}

Trapping efficiency was tested three times by spiking of a standard solution (10 LOQ) on the bottom of U-shaped glass tube connected with solid sorbent, and air was passed through the system at $60 \mathrm{~L} \cdot \mathrm{h}^{-1}$ for $4 \mathrm{~h}$ for trapping of vaporized acetamiprid. To help the volatilization of compounds, the U-shaped glass tube was heated to $70^{\circ} \mathrm{C}$. The residue in U-shaped glass tube and the amount trapped in solid sorbent were analyzed for mass balance. For breakthrough test, acetamiprid at 10 LOQ was spiked in the first part before passing air through the solid sorbent at $60 \mathrm{~L} \cdot \mathrm{h}^{-1}$ for $4 \mathrm{~h}$. Subsequently, first part and second part were analyzed separately. This test was repeated three times.

\section{Extraction of Acetamiprid from Exposure Matrices}

The dermal patches, gloves, masks, and socks were placed in a glass bottle $(100$ or $500 \mathrm{~mL})$ and extracted using 50 or 300 $\mathrm{mL}$ of methanol, depending on matrix size. Solid sorbent and glass fiber filter were placed into a $20 \mathrm{~mL}$ vial, and $10 \mathrm{~mL}$ of methanol was added. Those containers were shaken for $1 \mathrm{~h}$ in a shaker (Wooju Scientific, Gimpo, Korea) at room temperature. A sample of 1-2 $\mathrm{mL}$ of each extract was filtered through a $0.2 \mu \mathrm{m}$ pore syringe filter ( $4 \mathrm{~mm}$, Milipore, Billerica, MA, USA) before aliquots were analyzed using HPLC.

\section{Recovery (Matrix Extraction Efficiency) Test}

Three LOQ levels $(1,10$, and 100) of standard solution were spiked in dermal patches, gloves, socks, masks, glass fiber filter, and solid sorbent. Acetamiprid was extracted three times and analyzed according to the method described in the above analytical procedure.

\section{Field Trials and Sampling Procedure}

For exposure measurement during mixing/loading, only gloves were used and operators prepared the spray mixture by mixing acetamiprid WP with water after weighing a specific amount of powder. The mixture was stirred mechanically or by stick with hands on a speed sprayer (Model TLD ASS-555, Asia Motors, Daegu, Korea). After mixing/loading, gloves were removed for analysis. In the case of spraying, dermal patches
(Kim et al., 2012) were attached on 13 parts of body: head (1), front of neck (1), back of neck (1), chest/stomach (1), back (1), upper arm (2), forearm (2), thigh (2), and lower leg (2). And the operator wore cotton gloves, cotton socks, and mask (face exposure; $200 \mathrm{~cm}^{2}$ ). The spray mixture was applied to apple trees using a speed sprayer in field for 35-45 $\mathrm{min}$. The operator started spraying according to their usual technique, without other instructions. The application was carried out by driving the speed sprayer along the row. After the application, all materials were carefully removed avoiding accidental contamination of the different parts and then were separately placed in plastic bags.

PAM was used to monitor inhalation exposure. A glass fiber filter cassette and a solid sorbent were attached to the breathing zone with clips, and a personal air pump was fastened on waist with a by belt. After mixing/loading or spraying, the solid sorbent and filter were removed for analysis. The flow rate of personal air pump was $60 \mathrm{~L} \cdot \mathrm{h}^{-1}$.

\section{Calculation of Potential Dermal and Inhalation Exposure}

PDE per body part were calculated multiplying the unit amount $\left(\mu \mathrm{g} \cdot \mathrm{cm}^{-2}\right)$ of acetamiprid on dermal patch or mask with each surface area $\left(\mathrm{cm}^{2}\right)$ of body region for Korean, as suggested by Kim et al. (2011). The inhalation exposure rate $\left(\mu \mathrm{g} \cdot \mathrm{h}^{-1}\right)$ was obtained by dividing the inhalation exposure amount $(\mu \mathrm{g})$ by work time (h), and it was extrapolated to medium exercise $\left(1,270 \mathrm{~L} \cdot \mathrm{h}^{-1}\right)$ breathing rate for Korean male (Kim et al., 2011) to calculate potential inhalation exposure (PIE).

\section{Risk Assessment}

The risk assessment was carried out by modifying MOS formula in report of Hughes et al. (2008). The MOS was obtained as follows: $\mathrm{MOS}=\mathrm{AE} /[(\mathrm{PDE} \times \mathrm{AF})+\mathrm{PIE}] \times \mathrm{SF}$. Where AE (acceptable exposure $)=\operatorname{AOEL}\left(0.124 \mathrm{mg} \cdot \mathrm{kg}^{-1} \cdot \mathrm{d}^{-1}\right)$ $\times$ average body weight $(60 \mathrm{~kg}) ; \mathrm{PDE}=$ potential dermal exposure; $\mathrm{AF}=$ absorption factor; and $\mathrm{SF}=$ safety factor. The PDE and PIE values were obtained by the extrapolating of application time to $4 \mathrm{~h}$ for effective exposure per day. AF of 0.01 was used by the assumption of $10 \%$ of dermal absorption and $10 \%$ of cloth penetration. Thus, the actual formula used was: $\mathrm{MOS}=0.124 \times 60 /[(\mathrm{PDE} \times 0.01)+\mathrm{PIE}] \times 1$.

\section{Results and Discussion}

\section{Method Validation}

The analytical and sampling methods were fully validated through the various experiments as follows. LOQ was calculated as 4 times greater than LOD and the values were low enough for the detection of acetamiprid (Table 2). Good 
Table 2. Limit of detection (LOD), limit of quantitation (LOQ), reproducibility of analysis and linearity of calibration curve of acetamiprid.

\begin{tabular}{|c|c|c|c|c|c|c|}
\hline \multirow{2}{*}{ LOD } & \multirow{2}{*}{ LOQ } & \multicolumn{3}{|c|}{ Reproducibility (Area) } & \multirow{2}{*}{\multicolumn{2}{|c|}{ Linearity $\left(R^{2 y}\right)$}} \\
\hline & & LOQ level & Average & $\mathrm{CV}^{\mathrm{z}}(\%)$ & & \\
\hline \multirow{3}{*}{$0.25 \mathrm{ng}$} & \multirow{3}{*}{$1 \mathrm{ng}$} & 1 & 4.4 & 3.6 & Day of preparation & 1.0000 \\
\hline & & 10 & 46.8 & 1.0 & After 1 day & 0.9999 \\
\hline & & 50 & 238.6 & 0.9 & After 3 day & 0.9999 \\
\hline
\end{tabular}

${ }^{\mathrm{z}}$ Coefficient of variation.

${ }^{\mathrm{y}}$ Coefficient of determination.

Table 3. Recovery of acetamiprid from dermal patches, gloves, masks, socks, glass fiber filter, and solid sorbent.

\begin{tabular}{lcccccc}
\hline \hline \multirow{2}{*}{ Treated level } & \multicolumn{5}{c}{ Recovery $(\%)$} \\
\cline { 2 - 6 } & Dermal patch & Gloves & Mask & \multicolumn{1}{c}{ Socks } & Glass fiber filter & Solid sorbent \\
\hline LOQ & $108.5 \pm 2.2^{y}$ & $101.1 \pm 1.1$ & $98.9 \pm 6.5$ & $118.2 \pm 2.1$ & $95.9 \pm 1.7$ & $96.3 \pm 4.1$ \\
$10 \mathrm{LOQ}$ & $95.9 \pm 8.3$ & $88.8 \pm 6.9$ & $89.8 \pm 8.0$ & $94.8 \pm 9.1$ & $91.2 \pm 2.0$ & $85.3 \pm 5.1$ \\
$100 \mathrm{LOQ}$ & $103.9 \pm 6.7$ & $94.3 \pm 7.3$ & $94.3 \pm 8.0$ & $99.0 \pm 8.4$ & $90.9 \pm 6.2$ & $85.8 \pm 5.1$ \\
\hline
\end{tabular}

${ }^{\mathrm{Z}} \mathrm{LOQ}$ : limit of quantitation.

${ }^{\mathrm{y}}$ Mean $\pm \mathrm{SD}(\mathrm{n}=3)$.

Table 4. Trapping efficiency and breakthrough test of solid sorbent for acetamiprid.

\begin{tabular}{|c|c|c|}
\hline Test $^{z}$ & & \\
\hline \multirow{2}{*}{ Trapping efficiency } & Residue $^{y}$ & 96.4 \\
\hline & Trapped $^{\mathrm{x}}$ & 0 \\
\hline \multirow{2}{*}{ Breakthrough } & First part ${ }^{\mathrm{w}}$ & 93.8 \\
\hline & Second part ${ }^{v}$ & 0 \\
\hline
\end{tabular}

${ }^{\mathrm{z}}$ The tests were carried out at 10 limit of quantitation level.

${ }^{y}$ Amount left on the bottom of U-shaped tube.

${ }^{x}$ Amount trapped in solid sorbent.

${ }^{\mathrm{w}}$ First part of solid sorbent.

${ }^{v}$ Second part of solid sorbent.

reproducibility $(\mathrm{CV}<4 \%)$ showed that instrument was stable for analysis. Acetamiprid concentration and the response of the detector was linearly correlated $\left(\mathrm{R}^{2}>0.999\right)$ over the range of 0.1-10 $\mathrm{mg} \cdot \mathrm{L}^{-1}$ for 3 days. Recovery tests of acetamiprid from various sample matrices were conducted and ranged from 85.3 to $118.2 \%$ with the small relative standard deviation values (1.1-9.1), indicating the analytical procedures are reliable (Table 3). To validate the sampling method in inhalation exposure monitoring, trapping efficiency test and breakthrough test were performed. The trapping efficiency test was to measure the efficiency of solid sorbent for the trapping of pesticide in air. This experiment allowed a mass balance of over $96.4 \%$ by adding up the acetamiprid trapped in the solid sorbent and the residue at the bottom of the U-shaped glass tube. Most of acetamiprid remained at the bottom of the U-shaped glass tube because it was not volatile,. The breakthrough test evaluates the capacity of the solid sorbent to retain pesticide. The result of over $93.8 \%$ recovery from first part of resin without escaping to second part suggested that first part of resin has enough capacity to retain the corresponding amount of acetamiprid (Table 4). The validated approaches have been applied to assess potential exposure of the operator spraying acetamiprid in apple orchards.

\section{PDE and PIE}

In this study, cotton gloves were used for the measurement of potential dermal exposure to the operator's hands (Capri et al., 1999; Vercruysse et al., 1999) because they can be changed very quickly and that the provided some degree of safety for the operators, especially when using solid formulations (Tuomainen et al., 2002), even though exposure maybe overestimated if cotton is more absorbent than skin (Fenske et al., 1989). Only hands exposure were measured using gloves since the hands were reported to be significantly more exposed than the other body parts of the mixer and loaders (Kuye et al., 2007). Data for hands exposures amount during mixing/loading were expressed total amount $(\mu \mathrm{g})$ and not time rate, as this step was not time dependent (Hughes et al., 2006). Exposure amount of 
Table 5. Hands exposure during mixing/loading of acetamiprid.

\begin{tabular}{lcccc}
\hline \hline \multirow{2}{*}{ Operator } & \multicolumn{4}{c}{ Hands exposure $\left(\mathrm{mL} \cdot \mathrm{h}^{-1}\right)$} \\
\cline { 2 - 5 } & Minimum & Maximum & Average & Ratio to applied a. ${ }^{\mathrm{z}}(\%)$ \\
\hline 1 & 65.8 & 542.7 & 84.3 & $4.6 \times 10^{-4}$ \\
2 & 45.3 & 400.3 & 169.2 & $4.2 \times 10^{-4}$ \\
3 & 168.7 & 1132.1 & 649.3 & $1.6 \times 10^{-3}$ \\
4 & 32.9 & 327.8 & 147.8 & $3.7 \times 10^{-4}$ \\
\hline
\end{tabular}

${ }^{\mathrm{z}}$ Active ingredient.

Table 6. Dermal exposure of each body part during application of acetamiprid.

\begin{tabular}{|c|c|c|c|c|c|}
\hline \multirow{2}{*}{ Body part } & \multicolumn{5}{|c|}{ Dermal exposure $\left(\mathrm{mL} \cdot \mathrm{h}^{-1}\right)$} \\
\hline & Operator 1 & Operator 2 & Operator 3 & Operator 4 & Average \\
\hline Head & 58.6 & 62.8 & 44.2 & 35.8 & 50.3 \\
\hline Face & 5.8 & 22.1 & 7.7 & 2 & 9.4 \\
\hline Front of neck & 11.4 & 15 & 11 & 6.2 & 10.9 \\
\hline Back of neck & 9.9 & 7.1 & 5.3 & 3.4 & 6.4 \\
\hline Chest/stomach & 258.3 & 142.5 & 75.3 & 75 & 137.8 \\
\hline Back & 104.3 & 52.4 & 73.7 & 49.9 & 70.1 \\
\hline Left upper arm & 59.9 & 54.6 & 36 & 16.8 & 41.8 \\
\hline Right upper arm & 67.4 & 67.6 & 70.9 & 56.3 & 65.5 \\
\hline Left forearm & 53.6 & 43.8 & 41.1 & 15.4 & 38.5 \\
\hline Right forearm & 47.7 & 33.1 & 33 & 28.4 & 35.6 \\
\hline Hands & 36.1 & 38.5 & 16.8 & 28.3 & 29.9 \\
\hline Left thigh & 161.8 & 123.3 & 75.3 & 61.3 & 105.4 \\
\hline Right thigh & 177.3 & 131.6 & 101.8 & 67.4 & 119.5 \\
\hline Left lower leg & 102.9 & 65 & 42.7 & 52.6 & 65.8 \\
\hline Right lower leg & 75.9 & 66.2 & 54.1 & 35.5 & 57.9 \\
\hline Feet & 4.1 & 1 & 1.9 & 1.2 & 2.1 \\
\hline Total & 1235.1 & 926.6 & 690.5 & 535.3 & 846.9 \\
\hline Ratio to applied $a . i^{\mathrm{z}}(\%)$ & $8.0 \times 10^{-2}$ & $5.0 \times 10^{-2}$ & $3.0 \times 10^{-2}$ & $3.0 \times 10^{-2}$ & $5.0 \times 10^{-2}$ \\
\hline
\end{tabular}

${ }^{\mathrm{z}}$ Active ingredient.

hands during mixing/loading acetamiprid showed variable results (33-1,132 $\mu \mathrm{g})$ (Table 5). Exposure amount of operator 3 among 4 workers was noticeably high because he opened the WP package, rinsed the package in the water, and stirred it by stick while other workers opened WP package and mixed it with water by machine. The results also show that application habit can give rise to the high variability of the operator's exposure (Table 6). As shown in Table 6, the average PDE of acetamiprid during application ranged from 535 to $1,235 \mathrm{~mL} \cdot \mathrm{h}^{-1}$ which is corresponding to $0.03-0.08 \%$ of the applied amount. The level of exposure is known to be variable, depending on many different factors such as the type of crop, crop size, application technique, weather conditions, personal protective equipment, etc (Hughes et al., 2006; Machera et al., 2002; Tuomainen et al., 2002). In the cases of operator 1 and operator 2 , higher exposure rate was found than those of operator 3 and operator 4 . This result could be due to the narrower space between the trees and more windy condition when compared to other operators. Thigh was the most contaminated part with a rate of 224.9 $\mathrm{mL} \cdot \mathrm{h}^{-1}(26.6 \%)$ then the next was chest $(16.8 \%)$. In the same sense, when exposure was studied in apple orchard during application of methomyl, thighs and chest were identified as the most exposed body parts (Kim et al., 2012). Other majorly exposed parts were upper arms, forearms and lower legs. Head and face exposures were also relatively high. Inhalation exposure was monitored during the application and found to be around $10^{-8} \%$ of applied amount (Table 7 ). The highest inhalation exposure was observed with operator 2, probably due to relatively more windy weather during application. However, Cattani et al. (2001) reported that inhalation of chlorpyrifos was influenced by the ambient air temperature. 
Table 7. Inhalation exposure amount during application of acetamiprid.

\begin{tabular}{lccc}
\hline \hline \multirow{2}{*}{ Operator } & \multicolumn{3}{c}{ Inhalation exposure $(\mu \mathrm{g})$} \\
\cline { 2 - 4 } & Average & Ratio to applied a.i ${ }^{\mathrm{z}}(\%)$ & Ratio to dermal exposure $(\%)$ \\
\hline 1 & 0.009 & $2.3 \times 10^{-8}$ & $2.8 \times 10^{-5}$ \\
2 & 0.014 & $3.6 \times 10^{-8}$ & $6.9 \times 10^{-5}$ \\
3 & 0.006 & $1.5 \times 10^{-8}$ & $5.2 \times 10^{-5}$ \\
4 & 0.005 & $1.2 \times 10^{-8}$ & $4.0 \times 10^{-5}$ \\
\hline$z^{z}$ & &
\end{tabular}

${ }^{\mathrm{z}}$ Active ingredient.

Table 8. Calculation of margin of safety (MOS) for application of acetamiprid.

\begin{tabular}{lccr}
\hline \hline \multirow{2}{*}{ Operator } & \multicolumn{3}{c}{ Calculation of margin of safety } \\
\cline { 2 - 4 } & $\mathrm{PDE}^{\mathrm{z}}\left(\mathrm{mg} \cdot \mathrm{d}^{-1}\right)^{\mathrm{y}}$ & $\mathrm{PIE}^{\mathrm{x}}\left(\mu \mathrm{g} \cdot \mathrm{g}^{-1}\right)$ & $\mathrm{MOS}^{\mathrm{w}}$ \\
\hline 1 & 132.10 & 0.04 & 5.6 \\
2 & 83.92 & 0.06 & 8.9 \\
3 & 49.06 & 0.02 & 15.2 \\
4 & 50.11 & 0.02 & 14.8 \\
Average & 78.80 & 0.03 & 11.1 \\
\hline
\end{tabular}

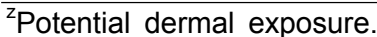

${ }^{\mathrm{y}}$ Including mixing/loading.

${ }^{x}$ Potential inhalation exposure.

${ }^{\mathrm{w}}$ Calculated as MOS $=0.124 \mathrm{mg} \cdot \mathrm{kg}^{-1} \mathrm{~d}^{-1} \times 60 \mathrm{~kg} /[(\mathrm{PDE} \times 0.01)+\mathrm{PIE}] \times 1$.

\section{MOS and Risk Assessment}

In risk assessment PDE values are considered together with the toxicological data for insecticides, then the resulting MOS values indicates that the spray procedure evaluated can be considered safe or not (Hughes et al., 2006). A value of MOS higher than 1 would indicate safe working conditions, whereas a MOS lower than 1 would mean unsafe conditions. In MOS calculation (Table 8), AE values are calculated on the basis of appropriate toxicological end point such as acceptable operator exposure level (AOEL) or no observed adverse effect level (NOAEL). If AOEL is available, NOAEL is not applicable and $\mathrm{SF}$ is 1 . AOEL was found to be $0.124 \mathrm{mg} \cdot \mathrm{kg}^{-1} \cdot \mathrm{d}^{-1}$ (EC, 2004). Average body weight for man is $60 \mathrm{~kg}$ (Choi et al., 2006). For dermal absorption, $10 \%$ of dermal absorption (Choi et al., 2006; Hughes et al., 2008; Kim et al., 2012; Ramos et al., 2010) and $10 \%$ of cloth penetration (Choi et al., 2006; Kim et al., 2012) were assumed, while $100 \%$ penetration was applied for inhalation (Kim et al., 2012; Oliveira and Machado-Neto, 2003). When no inhalation data was available, Huges et al. (2008) assumed a dermal exposure of $1 \%$. However, many study reported around from $10^{-4}$ to $10^{-2} \%$ levels of dermal exposure including this study (Capri et al., 1999; Kim et al., 2012; Machera et al., 2003; Oliveira and Machado-Neto, 2003). Calculated MOS values (5.6-15.2) was much higher than 1 in all cases, indicating that the working condition was of least risk.

\section{Literature Cited}

Capri, E., R. Alberici, C.R. Glass, G. Minuto, and M. Trevisan. 1999. Potential operator exposure to procymidone in greenhouses. J. Agr. Food. Chem. 47:4443-4449.

Cattani, M., K. Cena, J. Edwards, and D. Pisaniello. 2001. Potential dermal and inhalation exposure to chlorpyrifos in Australian pesticide workers. Ann. Occup. Hyg. 45:299-308.

Choi, H., J.K. Moon, K.H. Liu, H.W. Park, Y.B. Ihm, B.S. Park, and J.H. Kim. 2006. Risk assessment of human exposure to cypermethrin during treatment of mandarin fields. Arch. Environ. Contam. Toxicol. 50:437-442.

European Commission(EC). 2004. Acetamiprid SANCO/1392/2001-final. http://ec.europa.eu/food/ plant/protection/evaluation/newactive/ acetamiprid.pdf.

Fenske, R.A., S.G. Birnbaum, M.M. Methner, and R. Soto. 1989. Methods for assessing fieldworker hand exposure to pesticides during peach harvesting. Bull. Environ. Contam. Toxicol. 43:805-813

Hong, S.S., J.B. Lee, Y.K. Park, J.S. Shin, G.J. Im, and G.H. Ryu. 2007. The proposal for pesticide exposure estimation of Korean orchard farmer. Kor. J. Pest. Sci. 11:281-288.

Hughes, E.A., A.P. Flores, L.M. Ramos, A. Zalts, C.R. Glass, and J.M. Montserrat. 2008. Potential dermal exposure to deltamethrin and risk assessment for manual sprayers: Influence of crop type. Sci. Total. Environ. 391:34-40.

Hughes, E.A., A. Zalts, J.J. Ojeda, A.P. Flores, R.C. Glass, and J.M. Montserrat. 2006. Analytical method for assessing potential dermal exposure to captan, using whole body dosimetry, in small vegetable production units in Argentina. Pest. Manag. Sci. 62:811-818. 
Kim, E.H, H.R. Lee, H. Choi, J.K. Moon, S.S. Hong, M.H. Jeong, K.H. Park, H.M. Lee, and J.H. Kim. 2011. Methodology for quantitative monitoring of agricultural worker exposure to pesticides. Kor. J. Pest. Sci. 15:507-528.

Kim, E.H, J.K. Moon, H. Choi, S.M. Hong, D.H. Lee, H.M. Lee, and J.H. Kim. 2012. Exposure and risk assessment of insecticide methomyl for applicator during treatment on apple orchard. J. Kor. Soc. Appl. Biol. Chem. 55:95-100.

Korea Food and Drug Administration (KFDA). 2012. MRLs for pesticides in foods. KFDA, Osong, Korea.

Korean Statistical Information Service (KOSIS). 2011. Statistical database. http://kosis.kr/eng/database/database001000. jsp?listid= F\&subtitle=

Agriculture, Forestry And Fishery=Fruit production.

Kuye, R.A., K.J. Donham, S.P. Marquez, W.T. Sanderson, L.J. Fuortes, R.H. Rautiainen, M.L. Jones, and K.R. Culp. 2007. Pesticide handling and exposures among cotton farmers in the Gambia. J. Agromed. 12:57-69.

Machado-Neto, J.G. 2001. Determination of safe work time and exposure control need for pesticide applicators. Bull. Environ. Contam. Toxicol. 67:20-26.

Machera, K., E. Kapetanakis, A. Charistou, E. Goumenaki, and R.C. Glass. 2002. Evaluation of potential dermal exposure of pesticide spray operators in greenhouses by use of visible tracers. J. Environ. Sci. Heal. B. 37:113-121.
Machera, K., M. Goumenou, E. Kapetanakis, A. Kalamarakis, and R.C. Glass. 2003. Determination of potential dermal and inhalation operator exposure to malathion in greenhouses with the whole body dosimetry method. Ann. Occup. Hyg. 47:61-70.

Marin, A., J.L. Martinez Vidal, F.J. Egea Gonzalez, A. Garrido Frenich, C.R. Glass, and M. Sykes. 2004. Assessment of potential (inhalation and dermal) and actual exposure to acetamiprid by greenhouse applicators using liquid chromatography-tandem mass spectrometry. J. Chromatogr. B. 804:269-275.

Oliveira, M.L. and J.G. Machado-Neto. 2003. Use of manganese as tracer in the determination of respiratory exposure and relative importance of exposure routes in the safety of pesticide applicators in citrus orchards. Bull. Environ. Contam. Toxicol. 70:415-421.

Ramos, L.M., G.A. Querejeta, A.P. Flores, E.A. Hughes, A. Zalts, and J.M. Montserrat. 2010. Potential dermal exposure in greenhouses for manual sprayers: Analysis of the mix/load, application and re-entry stages. Sci. Total. Environ. 408:40624068.

Tuomainen, A., J.A. Kangas, W.J.A. Meuling, and R.C. Glass. 2002. Monitoring of pesticide applicators for potential dermal exposure to malathion and biomarkers in urine. Toxicol. Lett. 134:125-132.

Vercruysse, F., S. Drieghe, W. Steurbaut, and W. Dejonckheere. 1999. Exposure assessment of professional pesticide users during treatment of potato fields. Pest. Sci. 55:467-473. 